\title{
PACIENTES PORTADORES DE FISSURA LABIOPALATAL: UM ESTUDO RETROSPECTIVO
}

\author{
Luan Wesdley Ribeiro Maial \\ Juliana Campos Pinheiro" \\ Everton Freitas de Morais \\ Carlos Augusto Galvão Barboza IV \\ Bruno Torres Bezerra ${ }^{V}$ \\ Rafaella Bastos Leite
}

\section{RESUMO}

O objetivo desse estudo foi determinar o perfil epidemiológico de 115 pacientes portadores de fissura labiopalatal atendidos na SEAFESE (Sociedade Especializada em Atendimento ao Fissurado do Estado de Sergipe), em dois períodos distintos (Janeiro-Setembro de 2010/Janeiro-Setembro de 2015). Foram avaliados dados epidemiológicos como gênero, faixa etária, raça, procedência, renda familiar, hereditariedade, realização do exame pré-natal, presença de malformações congênitas, intercorrências durante a gestação, utilização de drogas e/ou medicamentos durante a gestação, condições de sanitarismo, contato com herbicidas e agrotóxicos, tipos de fissura e tratamento. O gênero feminino teve maior prevalência dos casos, com cerca de $55 \%$, faixa etária predominante $0-4$ anos (39\%), a raça parda foi a mais encontrada (48\%), sendo a maioria dos pacientes proveniente do interior do Estado (54\%). Grande parte dos pacientes apresentava baixa condição socioeconômica e o fator hereditariedade teve peso de $63 \%$. As más formações encontradas durante o estudo foram: surdez do ouvido esquerdo, autismo, alteração neurológica, ausência de 2 dedos pés/mãos, seis dedos nos pés, hidrocefalia, agenesia de antebraço e Síndrome de Appert. Apesar do alto índice de mães que realizaram o pré-natal, poucas foram comunicadas sobre a fissura durante a ultrassonografia. A frequência de tabagismo, exposição a herbicidas e agrotóxicos, consumo de ansiolíticos ou anticonvulsivantes foi baixa entre as mães. A fissura mais encontrada nos pacientes entrevistados foi a trans-forame incisivo.

PALAVRAS-CHAVE: Fenda palatina. Fenda labial. Anomalias Craniofaciais.

Cirurgião-dentista graduado pela Universidade Tiradentes ${ }^{\prime}$ ORCID: 0000-0003-1018-1311

Doutorando do Programa de Pós-Graduação em Ciências Odontológicas na UFRN. "I ORCID: 0000-0001-5687-7635

Doutorando do Programa de Pós-Graduação em Ciências Odontológicas na UFRN. ${ }^{\text {III }}$ ORCID: 0000-0002-2173-7672

Doutor em Patologia Oral e Professor do Programa de Pós-Graduação em Ciências IV Odontológicas UFRN. ORCID: 0000-0003-1979-9919

Doutorando em Ciências da Saúde pela Universidade Federal de Sergipe e Professor da $\mathrm{V}$ disciplina Estomatologia da Universidade Tiradentes. ORCID: 0000-0002-8830-9306

Docente da Faculdade Nova Esperança (Facene|Famene). ${ }^{\mathrm{VI}}$ ORCID: 0000-0002-3304-120X. Autor correspondente: rrafaella_bastos@hotmail.com 


\section{INTRODUÇÃO}

A fissura labiopalatina é uma deformidade congênita conhecida por sua etiologia multifatorial. $^{1}$ Desenvolve-se nas primeiras semanas de vida intrauterina, geralmente da quarta a oitava, com origem no aparelho branquial ou faríngeo e seus derivados. ${ }^{2}$ As fissuras atingem estruturas da face, tais como os ossos gnáticos, processos alveolares, além do lábio e do palato. Podem ser encontradas de forma isolada, em associação com outras alterações, ou como parte de síndromes. 3

Um aspecto relevante no tratamento do paciente fissurado é o seu acompanhamento precoce, o qual deve ser iniciado assim que o paciente for diagnosticado como portador de fissura labiopalatal. Em geral, procedimentos cirúrgicos são iniciados a partir dos três meses idade, quando o paciente, na maioria das vezes, possui peso adequado para ser submetido a cirurgia reparadora da fissura labial (queiloplastia). Dos 9 aos 18 meses de idade recomenda-se que seja submetido a cirurgia reparadora do palato duro (palatoplastia). Esses procedimentos cirúrgicos devem ser acompanhados e analisados por uma equipe interdisciplinar, composta por cirurgião buco-maxilo, cirurgião plástico, anestesiologista, ortodontista, fonoaudiólogo, psicólogo, enfermeiro, pediatra, cirurgião dentista clínico e nutricionista. Quanto mais precoce for iniciado o tratamento, mais

\section{MATERIAL E MÉTODOS}

Este estudo caracteriza-se por ser observacional, descritivo, transversal e epidemiológico de uma série de 115 pacientes, atendidos na SEAFESE em dois períodos distintos (Janeiro a Setembro de 2010 e Janeiro a Setembro de 2015). O presente estudo foi rápido será o ajustamento desse paciente à sociedade, visto que, indivíduos que apresentem essas deformidades faciais são discriminados e sofrem importantes problemas de origem psicológica. $3^{-6}$

No Brasil, um estudo pioneiro, realizado em escolas na cidade de Bauru-SP, encontrou uma prevalência de 1,54 casos para 1.000 pesquisados. ${ }^{4}$ Em Aracaju-SE, essa prevalência é de 0,54 casos para cada 1.000 nascidos vivos. 5 A Sociedade Especializada no Atendimento ao Fissurado do Estado de Sergipe (SEAFESE) está a 12 anos sendo a única no estado que presta esse tipo de serviço à sociedade. Parceira de hospitais e maternidades da capital e interior do Estado, pacientes são encaminhados ao serviço para avaliação multiprofissional e posterior tratamento clínico - cirúrgico e ainda acompanhamento realizado no Hospital São José, onde fica localizada a SEAFESE.

O objetivo desta pesquisa foi conhecer o perfil epidemiológico de uma série de 115 pacientes, portadores de fissura labiopalatal, atendidos na SEAFESE, para, com base nos resultados, serem criadas novas políticas de saúde junto aos órgãos representantes da saúde no Estado, bem como traçar campanhas preventivas e educativas para os pacientes portadores de fissura labiopalatal.

aprovado e protocolado pelo Comitê de Ética da UNIGRANRIO sob o $n^{\circ}$. 00540.317.000-09. Todos os pacientes foram examinados e os respectivos pais ou responsáveis submetidos a um questionário, em que foram analisadas as seguintes variantes: idade, raça, tipo de fis- 
sura, lado mais acometido, fatores genéticos, presença de algum tipo de anemia, intercorrências durante a gestação, utilização de drogas e/ou medicamentos durante a gestação, hábitos tabagistas, uso de ansiolíticos e/ou anticonvulsivantes, condições de sanitarismo, contato com herbicidas e/ou agrotóxicos, realização do exame pré-natal, quantidade de exame pré-natal feito, a ultrassonografia (US) e visualização da fissura labiopalatal na US.

\section{RESULTADOS}

No presente estudo, o gênero mais acometido pelas fissuras foi o gênero feminino, com $55 \%$ dos casos. Já o masculino apresentou

GRÁFICO 1- Distribuição dos pacientes por gênero
Foram excluídos da amostra pacientes que não apresentavam capacidade cognitiva para responder ao questionário, menores de idade, desacompanhados de responsáveis legais e pacientes que, por algum motivo, não quiseram participar da pesquisa.

Todos os dados foram tabulados nos programas Word e Excel (2013) e tiveram seus dados representados através de gráficos e tabelas.
$45 \%$ dos pacientes, conforme observado no gráfico 1.

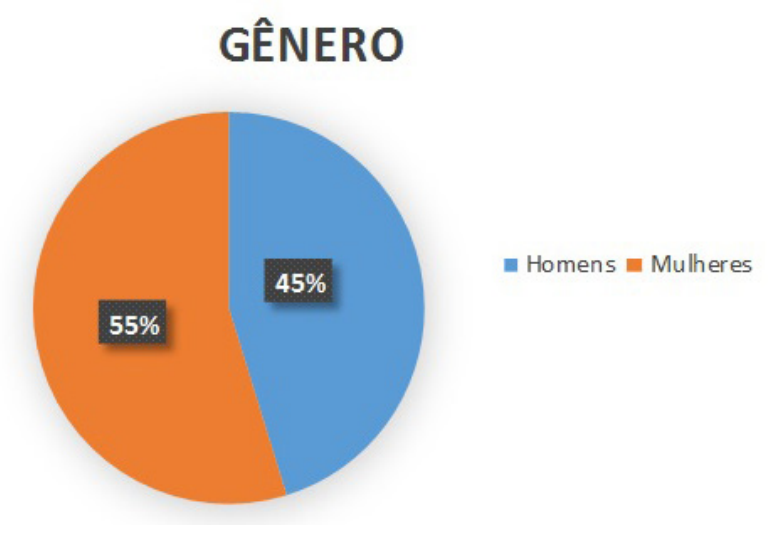

Com relação à faixa etária dos pacientes entrevistados no estudo, o grupo etário de 0-4 anos foi o mais acometido com 39\% dos casos (45 pacientes), seguido com $15 \%$ os pacientes acima de 25 anos de idade (17 pacientes); 5-9 anos com $14 \%$ (16 pacientes); $15-19$ anos com 13\% (15 pacientes); 10-14 anos com 11\% (13 pacientes) e por último a faixa etária de 20-24 anos com $8 \%$ (9 pacientes) podendo ser observado gráfico 2. 
GRÁFICO 2- Distribuição dos pacientes por faixa etária

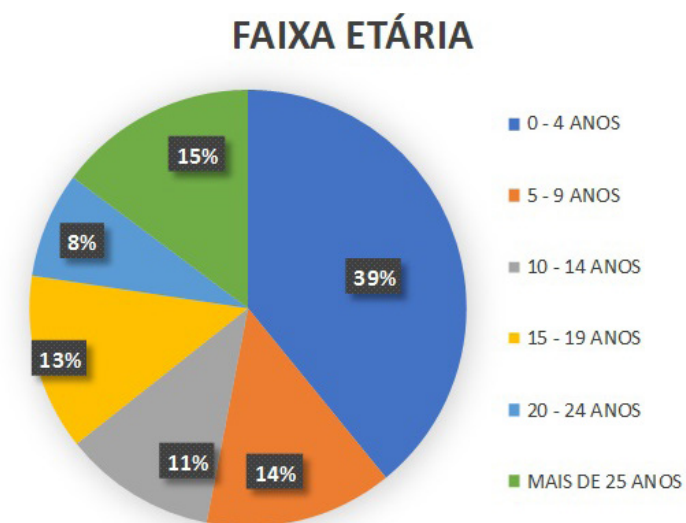

O gráfico 3 apresenta os resultados em relação à raça e constata-se que a cor parda foi predominante entre os pacientes entrevistados, com cerca de $48 \%$ dos casos

GRÁFICO 3- Distribuição dos pacientes por raça
(55 pacientes), seguidos dos brancos com 40\% (46 pacientes); negros com 11\% (12 pacientes); indígena $1 \%$ (1 paciente).

\section{RAÇA}

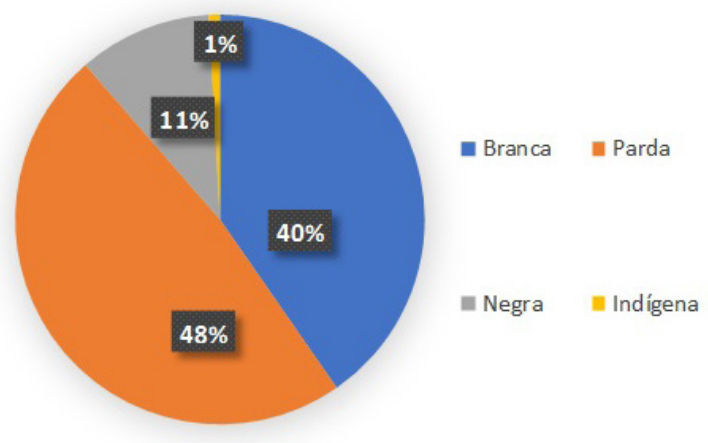

Quanto à procedência dos pacientes, cientes); outros Estados 8\% (9 pacientes). Re$54 \%$ eram provenientes do interior do estado sultados podem ser observados no gráfico 4 . (62 pacientes); $38 \%$ da capital Aracaju (44 pa-

GRÁFICO 4- Distribuição dos pacientes por procedência 
GRÁFICO 4- Distribuição dos pacientes por procedência

\section{PROCEDÊNCIA}

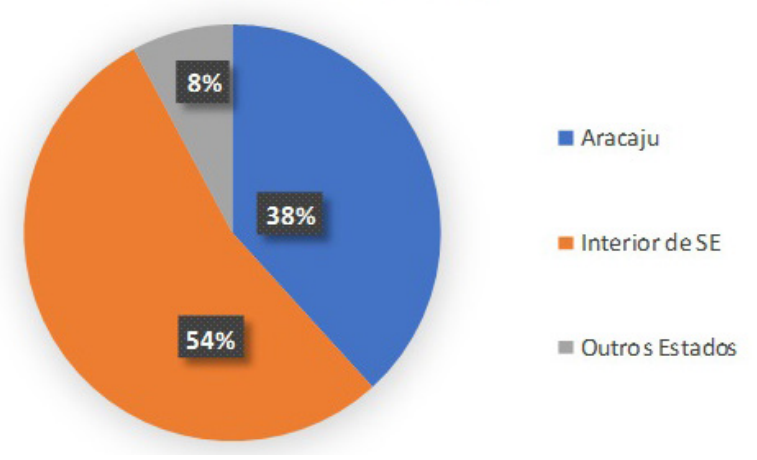

Os gráficos 5 e 6 apresentam os resul-

a pré-forame com 27 pacientes. As fissuras ratados em relação aos tipos de fissura. A fissuras de face foram diagnosticadas em apenas ra trans-forame incisivo apresentou o maior 1 paciente durante os períodos de coleta de número de casos, com 52 pacientes; seguida dados.

pela pós-forame incisivo, com 35 pacientes, e

GRÁFICO 5- Distribuição dos pacientes quanto ao tipo de fissura

\section{TIPO DE FISSURA}

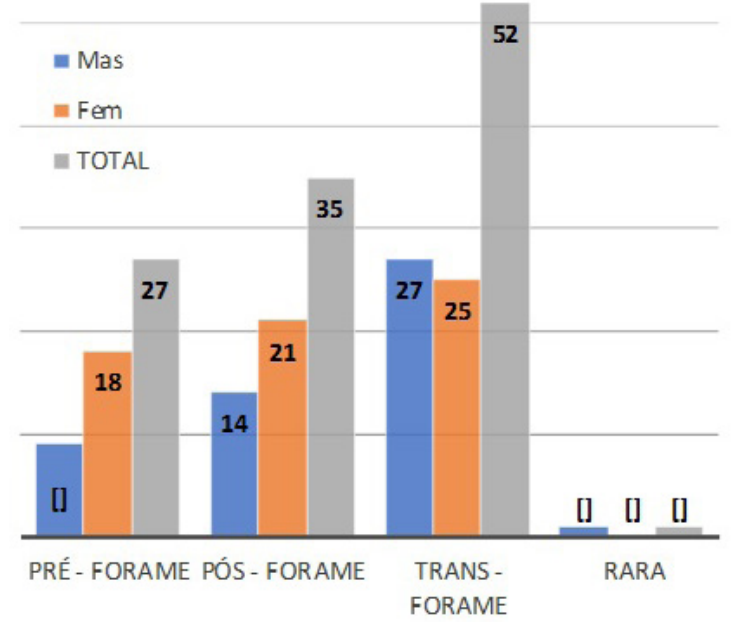


GRÁFICO 6- Distribuição dos pacientes quanto ao tipo de fissura

\section{LADO MAIS ACOMETIDO}

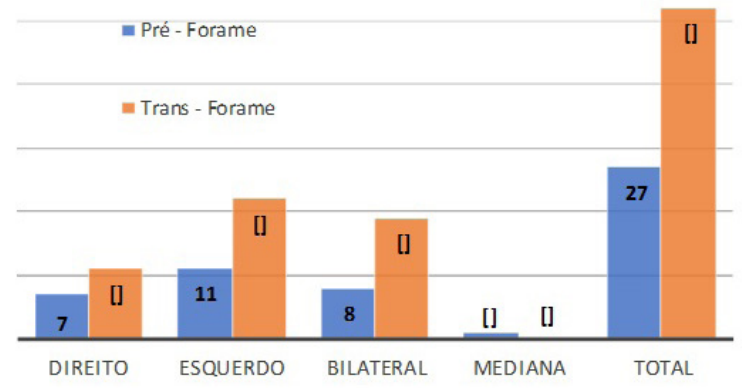

Das 27 fissuras pré-forame incisivo, 7 eram unilaterais direita, 11 unilaterais esquerdas e 8 bilaterais; 1 mediana, entre as 52 trans-forame incisivo encontradas, 11 eram unilaterais diretas, 22 unilaterais esquerdas e 19 bilaterais. Resultados podem ser observados no gráfico 5 e 6 .

Quanto a renda familiar apenas duas categorias foram obtidas. Ou seja, $60 \%$ dos pacientes apresentavam renda familiar de 1 a 4 salários mínimos (SM) e 40\% dos pacientes menos de $1 \mathrm{SM}$. As outras categorias não foram encontradas no nosso estudo (4 a $10 \mathrm{SM}$ e mais de $10 \mathrm{SM}$ ).

Com relação à hereditariedade, 37\% dos pacientes relataram ter na família algum caso de fissura labiopalatal, $63 \%$ afirmaram ser o primeiro portador de fissura labiopalatal em suas famílias.

Quanto à realização do exame pré-natal, $79 \%$ das mães relatam ter feito o exame. Em relação a US, 75\% das mães realizaram, mas apenas $8 \%$ das mães foram comunicadas, durante o exame, sobre a fissura labiopalatal.

As más formações congênitas estavam presentes em $8 \%$ dos pacientes estudados, e $92 \%$ dele não apresentavam qualquer tipo de má formação associada à fissura labiopalatal.
Do total de mães entrevistadas nesse estudo, $16 \%$ relataram que houve intercorrências durante a gestação, enquanto $84 \%$ relataram não ter tido qualquer intercorrência no período gestacional.

No que se refere ao comportamento das mães, durante a gestação, $6 \%$ relataram ter feito uso de algum tipo de droga, quando $94 \%$ negou a utilização desses produtos. Apenas $2 \%$ utilizaram ansiolítico ou anticonvulsivante. Quanto aos hábitos tabagistas, $14 \%$ das mães afirmaram ter feito uso de fumo, durante a gestação, e $86 \%$ negaram a utilização do fumo.

Das condições de sanitarismo, $68 \%$ apresentavam água encanada e moravam em ruas asfaltadas, enquanto $31 \%$ não tinham as mesmas condições. Já $1 \%$ relatou não saber.

A SEAFESE segue um protocolo quando se trata da realização de procedimentos cirúrgicos na reabilitação dos pacientes portadores de fissura labiopalatal. Grande número de pacientes da amostra recebeu tratamento na SEAFESE, sendo 47 queiloplastias realizadas pelo serviço, e 43 palatoplastias. Em alguns casos esses procedimentos ocorreram no mesmo ato operatório. Dois pacientes realizaram cirurgias ortognáticas. Quinze pacientes não haviam realizado qualquer tipo de pro- 
cedimento cirúrgico, pois ainda não estavam aptos em relação a idade e peso, ou deram

\section{DISCUSSÃO}

As fissuras labiopalatais são conceituadas com alterações faciais de origem embrionária, resultante da falta de fusão dos processos nasais mediais entre si, e desses com os processos maxilares. ${ }^{6-10}$

Dos 115 pacientes com fissura labiopalatal, $55 \%$ dos casos eram do sexo feminino, semelhante aos dados encontrados por Altman7; Loffredo et $\mathrm{al}^{11}$. Esses resultados discordam dos achados de ${ }^{4,12}$, em que o sexo feminino é o menos acometido, pelo fato do fechamento da fenda palatina no estado embrionário ser mais precoce no gênero feminino que no masculino.

Em relação a faixa etária dos pacientes entrevistados, o grupo etário de 0-4 anos foi o mais frequente com $39 \%$ dos casos ( 45 pacientes). Esse elevado número de pacientes com pouca idade, deve-se a divulgação que a SEAFESE desempenha. Durante todos esses anos em Sergipe, sempre trabalhando em parceria com hospitais e maternidades, constata-se que o atendimento a pacientes fissurados com pouca idade tem crescido a cada ano. Em relação a faixa etária, 15\% dos casos apresentaram pacientes acima de 25 anos de idade (17 pacientes); 5-9 anos com 14\% (16 pacientes); 15 -19 anos com 13\% (15 pacientes); 10-14 anos com 11\% (13 pacientes) e por último a faixa etária de 20-24 anos com $8 \%$ (9 pacientes). Dados que podem ser comparados aos de Di Ninno et al13.

Dos pacientes submetidos ao questionário, a raça prevalente foi a parda, com cerca de $48 \%$ dos casos. Dados diferentes aos encontrado por da Costa et al ${ }^{14}$. Em relação a proce- entrada no serviço recentemente, e estavam passando pelas primeiras avaliações. dência, a maioria dos pacientes são provenientes do interior do Estado de Sergipe, cerca de 54\% dos entrevistados. Dados semelhantes aos encontrados por Bezerra. 5

A fissura trans-forame incisivo apresentou o maior número de casos, com cerca de 52 pacientes, seguida da pós-forame e pré-forame, respectivamente. Apenas uma fissura rara de face foi detectada durante o período de coleta de dados, de acordo com Di Ninno et $\mathrm{al}^{13}$; Costa et al ${ }^{14}$. As fissuras (pré-forame e pós-forame) apresentam incidência no sexo feminino, como descrito por Altman. 7

Quanto ao lado acometido, obtivemos: 27 fissuras pré-forame incisivo, das quais 7 eram unilaterais direita, 11 unilaterais esquerda e 8 bilaterais. Entre as 52 trans-forame incisivo encontradas, 11 eram unilaterais diretas, 22 unilaterais esquerdas e 19 bilaterais.

Em relação a renda familiar, $60 \%$ dos pacientes apresentavam ganhos de 1 a 4 salários mínimos (SM), mensalmente, e o restante dos pacientes menos de 1 salário mínimo. No quesito hereditariedade, $37 \%$ dos pacientes têm outros casos de fissura na família e $63 \%$ dizem que foram os primeiros a apresentar. A história intrafamiliar parece ser um aspecto importante na etiologia da fissura, pois pode ser um fator de suma importância para que surjam outros casos na família desses pacientes. Dados encontrados são semelhantes aos encontrados por Altman.7 Entretanto, vários fatores devem ser levados em consideração quando um paciente é diagnosticado com esse tipo de má formação, o que torna inconclusivo a teoria da geneticidade apenas. 
Quanto a realização da US, 75\% das mães relataram ter realizado o exame, mas apenas 9\% foram informadas durante o procedimento sobre a presença de fissura. Esses baixos índices de visualização, durante a US, devem-se ao fato de dificuldade inerente as fissuras em serem visualizadas durante o exame, a falta de experiência por parte do profissional que realiza o exame e baixa qualidade dos aparelhos de US utilizados para realização da maioria dos exames. Em relação a má formação congênita, apenas $8 \%$ dos pacientes as apresentaram e $92 \%$ não mostraram qualquer tipo de má formação associada a fissura.

\title{
CONCLUSÃO
}

O presente estudo demonstra que, mediante os dados obtidos, chegou-se as seguintes constatações: o sexo feminino foi o mais acometido; o grupo etário de 0-4 anos o mais afetado; a cor parda é predominante entre os pacientes entrevistados; a maioria dos pacientes é procedente do interior do Estado de Sergipe; a fissura trans-forame foi a prevalente; a maior parte foi de fissuras unilaterais esquerdas. A maioria dos pacientes tinha renda familiar de 1 a 4 SM; a relação de parentesco mais encontrada foi entre primos; a maior parte das mães realizou o pré-natal e US, mas em grande parte não houve a visualização da anormalidade. Poucos casos de intercorrências, durante a gestação, foram encontrados e baixo índice de mães fizeram uso de ansiolíticos e/ou anticonvulsivantes. É de suma importância que estudos como estes sejam realizados a fim de serem criadas novas políticas e campanhas de promoção e prevenção de saúde dos pacientes portadores de fissuras labiopalatais.

\section{PATIENTS WITH CLEFT LIP AND PALATE: A RETROSPECTIVE STUDY}

\begin{abstract}
The objective of this study was to determine the epidemiological profile of 115 patients with cleft lip and palate treated at SEAFESE (Cleft Patient Specialized Society of Sergipe), in two periods (Jan-Sep, 2011/Jan-Sep 2015). We evaluated epidemiology data such as gender, age, race, origin, family income, inheritance, realization of prenatal examination, presence of congenital malformations, complications during pregnancy, use of drugs and / or drugs during pregnancy, sanitary conditions, contact with herbicides and pesticides, cleft types and treatment. the females have a higher prevalence of cases, with around $55 \%$, predominant age group 0-4 years (39\%), the brown race was the most frequent (48\%), with most coming from the state patients (54\%). Most patients have low socioeconomic status, heredity $63 \%$. The malformations found during the study were deafness of the left ear, autism, neurological changes, and absence of 2 feet/hands fingers, six toes, hydrocephalus, agenesis forearm and Appert syndrome. Despite the high number of mothers who underwent prenatal, little has been reported on the cleft during the ultrasound. The frequency of smoking, exposure to herbicides and pesticides, and consumption of tranquilizers or anticonvulsants was low among mothers of patients with
\end{abstract}


cleft. The fissure most often found in patients interviewed was the trans-foramen incisive.

KEYWORDS: Cleft palate; Cleft lip; Craniofacial abnormalities.

\section{REFERÊNCIAS}

1. Veronez FS, Tavano LD. Modificações psicossociais observadas pós-cirurgia ortognática em pacientes com e sem fissuras labiopalatinas. Arq Cienc Saude. 2005;12:133-7.

2. Borges-Osório MR, Robinson WM. Genética Humana. $2^{\mathrm{a}}$ ed. Porto Alegre: Artmed; 2001.

3. Gorlin R, Cohen M, Hannekam R. Syndromes of the head and neck. 4th ed. New York:Oxford University Press; 2001.

4. Nagem Filho H, Moraes N, Rocha RGF. Contribuição para o estudo da prevalência das mas formações congênitas labio- -palatais na população escolar de Bauru. Rev. Fac. Odontologia São Paulo. 1968;6:111-28.

5. Melgaço CA, DI Ninno CQMS, Penna LM et al. Aspec ᄀtos ortodônticos /Ortopédicos e Fonoaudiológicos relacionado a pacien-tes portadores de fissuras labiopalatinas. J. Bras. Ortodon. Ortop. Facial. 2002; 7 (37): 23-32.

6. Altmann EBC. Fissuras labiopalatinas. 4. ed. Carapicuíba-SP: Ed. Pró-Fono R. Atual Cient. 1997.

7. Al Omari F, Al-Omari IK. Cleft lip and palate in Jordan: birth prevalence rate. Cleft Palate Craniofac J. 2004;41:609-612.
8. Bellis $\mathrm{TH}$, Wohlgemunth $\mathrm{B}$. The incidence of cleft lip and palate deformities in the Southeast of Scotland (1971-1990). J Orthod.1999;26:121-125.

9. Baroneza JE, Faria MJSS, Kuasne H. et al. Dados epindemiológicos de portadores de fissuras labiopalatinas de uma instituição especializada de Londrina, Estado do Paraná. Acta Sci Health Sci. 2005; 27 (1): 31-5.

10. Loffredo LCM, Freitas JAS, Grigolli AAG. Prevalências de fissuras orais de 1975 a 1994. Rev. Saude Publica. 1994; 35(6): 371-5.

11. Collares MVM, Westphalen ACA, Costa TCD, Goldim Jr. Fissuras lábio-palatinas: incidência e prevalência da patologiano Hospintal de Clínicas de Porto Alegre. Um estudo de 10 anos. Rev AMRIGS. 1995;39(3):183.

12. Di ninno cqms et al. Levantamento epidemiológico dos pacienntes portadores de fissura de lábio e/ou palato de um centro especializado de Belo Horizonte. Rev. CEFAC. 2011; 13 (6): 1002-8.

13. Da Costa RR, Takeshita WM, Farah GJ. Levantamento epidemiológico de fissuras labiopalatais no município de Maringá e região. Rev. Paulista Cir Dent 2013;67(1):40-4. 